\title{
Combination of IL-12 gene therapy and CTX chemotherapy inhibits growth of primary B16(F10) melanoma tumors in mice
}

\author{
Iwona Mitrus, Klaudia Delić, Natalia Wróbel, Ewa Missol-Kolka and Stanisław Szala ${ }^{\bowtie}$ \\ Department of Molecular Biology, Maria Skłodowska-Curie Memorial Cancer Center and Institute of Oncology, \\ Gliwice Branch, Gliwice, Poland; ${ }^{\circledR}$-mail: sszala@io.gliwice.pl
}

Received: 22 March, 2005; revised: 05 October, 2005; accepted: 25 October, 2005

available on-line: 29 May, 2006

\begin{abstract}
We investigated suppression of murine B16(F10) melanoma tumor growth following a therapy which involved concomitant administration of cyclophosphamide and plasmid DNA bearing interleukin-12 gene. Since both therapeutic factors display antiangiogenic capabilities, we assumed that their use in blocking the formation of new blood vessels would result in augmented inhibition of tumor growth. This combined therapy regimen indeed resulted in a considerable suppression of tumor growth. We observed a statistically significant extension of treated animals' lifespan. Interestingly, the therapeutic effect was also obtained using a plasmid without an interleukin gene insert. This observation suggests that plasmid DNA, which has been widely applied for treating neoplastic tumors, contains element(s) that elicit immune response in mice.
\end{abstract}

Keywords: interleukin-12 gene, antiangiogenic therapy, combined therapy

\section{INTRODUCTION}

Formation of new blood vessels (angiogenesis) is critical for continued growth of neoplastic tumors (Folkman, 1971). Since tumor vasculature assures the supply of necessary nutrients and oxygen, inhibiting its formation should lead to the arrest of tumor growth and spread (Longo et al., 2002; Indraccolo, 2004). The quest for effective inhibitors of neoangiogenesis has been one of the most intensely explored anticancer research strategies in recent years. Such inhibition can ensue from the action of various factors, such as endogenous proteinaceous inhibitors, exogenous compounds, gene therapy constructs as well as chemotherapeutics (Longo et al., 2002; Sivridis et al., 2003; Indracollo, 2004; Tandle et al., 2004). In this report, we show data demonstrating that suppression of tumor growth can result from a combination of two therapeutic agents: a genetic construct in the form of plasmid DNA containing interleukin-12 (IL-12) gene and a chemotherapeutic, cyclophosphamide (CTX).

IL-12 is a 72-kDa cytokine, consisting of two subunits: p45 and p35 (Hendrzak \& Brunda, 1995).
This cytokine stimulates proliferation of $\mathrm{T}$ lymphocytes and NK cells, induces synthesis of other cytokines (among them INF- $\gamma$ and TNF- $\alpha$ ) and displays antiangiogenic properties (Hendrzak \& Brunda, 1995; Tandle et al., 2004).

Compounds employed primarily as chemotherapeutics can also be administered as antiangiogenic agents (Browder et al., 2000; Longo et al., 2002; Kerbel \& Kamen, 2004). They induce primarily apoptosis of endothelial cells within the vascular bed of a tumor. This, in turn, leads to apoptosis of tumor cells (Browder et al., 2000). Drugs of this kind are frequently dispensed in low doses (so-called metronomic schedule). Such scheduling of chemotherapeutics minimizes their toxicity (Longo et al., 2002). Since combining independent antiangiogenic agents may lead to synergy it appeared to us that the strategy of supplementing gene therapy with chemotherapy would perhaps improve the effectiveness of treatment of tumors. We here demonstrate that gene therapy based on an IL-12-containing plasmid construct and accompanied by chemotherapy with CTX results in significant suppression of B16(F10) tumor growth in mice. 


\section{MATERIALS AND METHODS}

Plasmids. We used in our therapeutic experiments the pBCMGSNeo/mIL-12 plasmid that contains sequences encoding both subunits of murine IL-12 (p35 and 540, separated by an IRES sequence), under the control of the CMV promoter (construct obtained from Dr. H. Yamamoto from Osaka University, Japan). As a control, the pBCMGSNeo plasmid (obtained from Dr. H. Karasuyama from the former Basel Institute of Immunology, Switzerland) was used. For in vivo experiments plasmids were isolated using EndoFree Giga Kit (Qiagen). Mice received intratumorally, for 2 days, $50 \mu \mathrm{g}$ of plasmid DNA in $100 \mu 1$ Ringer's solution with $0.05 \%$ lactose.

Cell culture. B16(F10) murine melanoma cell line was cultured in RPMI 1640 medium supplemented with $10 \%$ fetal bovine serum (FBS) (ICN), at $37^{\circ} \mathrm{C}$ under $5 \% \mathrm{CO}_{2}$.

Animals. Six- to eight-week-old C57BL/6 mice were bred and maintained at an on-site animal facility.

B16(F10) tumors. Female C57BL/6 mice had their dorsal side shaved and were inoculated subcutaneously $\left(2 \times 10^{5}\right.$ B16(F10) melanoma cells in $100 \mu 1$ PBS $^{-}$per animal). Administration of therapeutic DNA was started on day 8 of experiment, when tumors were $4-5 \mathrm{~mm}$ in diameter. The size of the tumors was calculated from the following formula: Volume $=(\text { Width })^{2} \times$ Length $\times 0.52$.

Cyclophosphamide (CTX). (Baxter Oncology $\mathrm{GmbH}$ ) was injected into mice intraperitoneally $(170 \mathrm{mg} / \mathrm{kg}$, in $0.9 \% \mathrm{NaCl}$ ) every 6 days (Browder et al., 2002).

IL-12 determination in tumor tissue. Plasmid DNA $(50 \mu \mathrm{g})$ in $100 \mu \mathrm{l}$ of Ringer's solution was administered into neoplastic tumors $(6-7 \mathrm{~mm})$ once. After 3, 6, 9 or $24 \mathrm{~h}$ the tumors were excised and homogenized in lysis buffer (Promega) supplemented with $1 \mathrm{mM}$ EDTA ( $\mathrm{pH} \mathrm{8.0)}$ as well as protease inhibitors (1 mM PMSF and $1 \mathrm{mM}$ benzamidine). IL-12 concentration in homogenates was determined using Mouse Quantikine M IL-12 p70 kit (R\&D Systems).

\section{RESULTS AND DISCUSSION}

This short report summarizes the results of B16(F10) murine melanoma therapy achieved using a combination of two agents: a plasmid construct containing an IL-12 gene insert (genetic therapy) and CTX (chemotherapy).

Plasmid DNA carrying cloned IL-12 gene was injected intratumorally without the use of a carrier. Two different administration schedules (twice a week or every two days) were evaluated. The more frequent administration of the IL-12 insert-containing plasmid resulted in improved therapeutic efficiency. Possibly, this was the consequence of the very brief duration (6-9 h) of the IL-12 residence in the tumor (Fig. 1). Within $24 \mathrm{~h}$ of DNA administration, the amount of this cytokine drops down to levels comparable to those in a tumor not subjected to the treatment. An increase of the IL-12 level was also noted following administration of a control plasmid (without insert), but in this case the cytokine was present for no longer than $6 \mathrm{~h}$. This transitory occurrence probably represents an immune response due to the presence of $\mathrm{CpG}$ sequences in plasmid DNA (Carpentier et al., 2003).

Some authors reported (Budryk et al., 2000; Shi et al., 2002) that IL-12 gene administration yielded total regression of Renca (murine renal carcinoma) and CT26 (murine adenocarcinoma) tumors. In our study we could not achieve a complete tumor eradication. This might be because we examined a different tumor model which is less immunogenic than either CT26 or Renca.

As a chemotherapeutic, we used CTX. Initially, administration schedules were analogous with those adopted by Browder et al. (2000). The observed side effects were, however, much stronger than those reported therein. If drug administration was repeated more than four times, the animals' body mass started to decrease and even some mice harboring small tumors died. This was especially striking in cases where the combined therapy regimen was applied. When using DNA, we were constrained to limit the application of CTX to three doses and we decided to administer the therapeutic factors intermittently. This treatment limited body weight loss. Typically, the therapy was initiated by injecting the

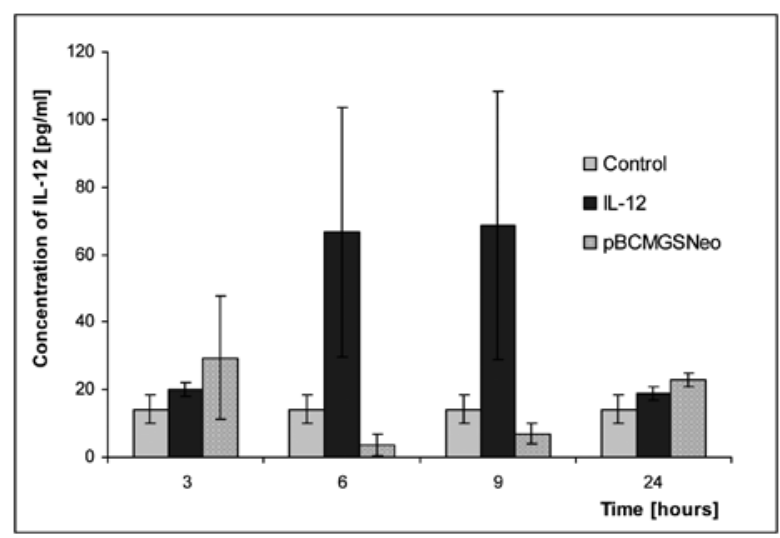

Figure 1. Determination of IL-12 level in tumors.

The experiments were carried out on mice harboring tumors 5-6 mm in diameter. IL-12 concentration was assessed following single administration of $50 \mu \mathrm{g}$ of DNA in $100 \mu \mathrm{l}$ Ringer's solution with $0.05 \%$ lactose. Tumors were excised after 3, 6, 9 or $24 \mathrm{~h}$. Each data point represents an average of three determinations. IL-12 concentration is expressed in pg per $\mathrm{ml}$ of tissue homogenate. As control, tissue from untreated tumor was used. 


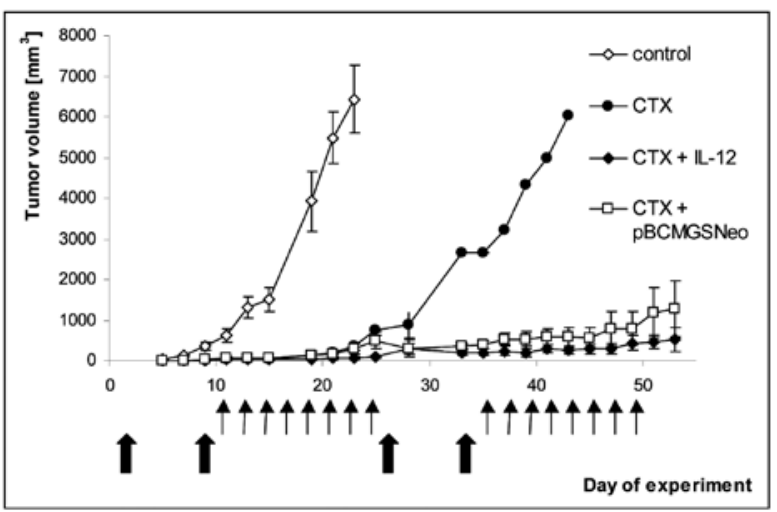

Figure 2. Suppression of tumor growth following administration of CTX and IL-12.

C57Bl/6 mice were inoculated subcutaneously with B16(F10) cell suspension. CTX was administered intraperitoneally (170 mg per kilogram of body mass; large arrows). DNA (empty pBCMGSNeo plasmid or pBCMGSNeo with IL-12 gene insert) was administered intratumorally $(50 \mu \mathrm{g} /$ $100 \mu \mathrm{l}$ of Ringer's solution supplemented with $0.05 \%$ lactose; small arrows). Eight mice were used per experimental and control group each.

animals with CTX on the 3rd day after inoculation with neoplastic cells. A postponed start of the therapy (after mice had already developed identifiable tumors) yielded much worse therapeutic effects (not published). This observation agrees with the earlier data published by Budryk et al. (2000).

In our experiments which tested a combination therapy the animals first received two doses of the chemotherapeutic agent on days 3 and 9 of therapy. Then, plasmid DNA administration (either empty vector or vector containing IL-12 insert) ensued from day 11 to day 25. Following this, on days 27 and 33 the mice again received the chemotherapeutic and the therapy was concluded with plasmid DNA readministration between days 35 and 49. Upon premature termination of therapy, tumors quickly regrew and animal survival was reduced as compared to that in full-length therapy.

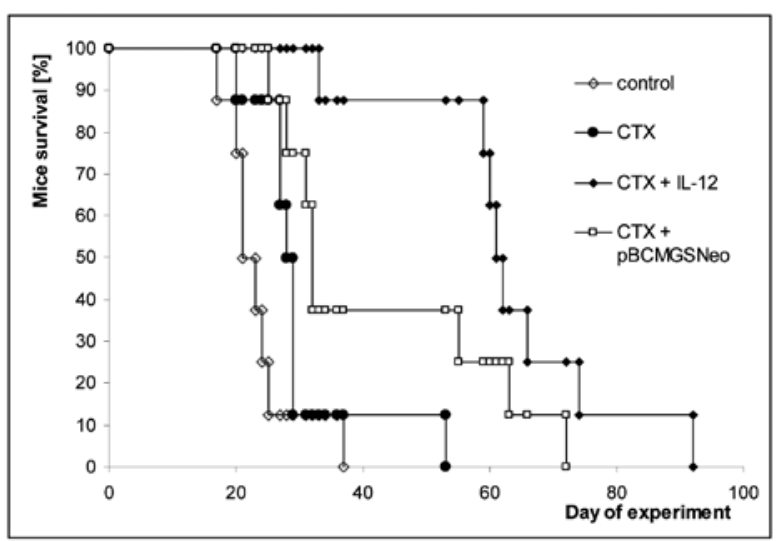

Figure 3. Survival of mice subjected to combined therapy.
Administration of CTX alone only yielded a minor therapeutic effect. Although the rate of tumor growth was diminished, the survival of treated animals did not differ significantly from that of control mice.

In the case of combined therapy used by us the rate of tumor growth was decidedly lower compared to that in mice receiving CTX only (Fig. 2). To explain this effect, one should consider immune response mechanisms. Plasmid DNA contains unmethylated CpG sequences, which are known to induce expression of proinflammatory cytokines at the tumor site (Carpentier et al., 2003). For the empty plasmid the effect was weaker compared to plasmid DNA containing the IL-12 insert. Nonetheless, also in this case suppression of tumor growth was observed and animal survival was better than that of mice treated with CTX only (Fig. 3).

To conclude: a combined therapy using an appropriate administration schedule of an IL-12 gene construct and CTX distinctly reduces the rate of murine melanoma tumor growth and prolongs animal survival.

\section{Acknowledgements}

We thank Dr. Aleksander Sochanik for reviewing the article.

This work was supported by grant from the State Committee for Scientific Research (KBN, Poland) No. 6 P05A 06221.

\section{REFERENCES}

Browder T, Butterfield CE, Kraling BM, Shi B, Marshall B, O'Reilly MS, Folkman J (2000) Antiangiogenic scheduling of chemotherapy improves efficacy against experimental drug-resistant cancer. Cancer Res 60: 1878-1886.

Budryk M, Wilczynska U, Szary J, Szala S (2000) Direct transfer of IL-12 gene into growing Renca tumors. Acta Biochim Polon 47: 385-391.

Carpentier AF, Auf G, Delattre JY (2003) CpG-oligonucleotides for cancer immunotherapy: review of the literature and potential applications in malignant glioma. Front Biosci 8: e115-127.

Folkman J (1971) Tumor angiogenesis: therapeutic implications. N Engl J Med 285: 1182-1186.

Hendrzak JA, Brunda MJ (1995) Interleukin-12. Biologic activity, therapeutic utility, and role in disease. Lab Invest 72: 619-637.

Indraccolo S (2004) Undermining tumor angiogenesis by gene therapy: an emerging field. Curr Gene Ther 4: 297-308.

Kerbel RS, Kamen BA (2004) The anti-angiogenic basis of metronomic chemotherapy. Nat Rev Cancer 4: 423-436.

Longo R, Sarmiento R, Fanelli M, Capaccetti B, Gattuso D, Gasparini G (2002) Anti-angiogenic therapy: rationale, challenges and clinical studies. Angiogenesis 5: 237-256.

Shi F, Rakhmilevich AL, Heise CP, Oshikawa K, Sondel PM, Yang NS, Mahvi DM (2002) Intratumoral injection of interleukin-12 plasmid DNA, either naked or 
in complex with cationic lipid, results in similar tumor regression in a murine model. Mol Cancer Ther 1: 949-957.

Sivridis E, Giatromanolaki A, Koukourakis MI (2003) The vascular network of tumours - what is it not for? J Pathol 201: 173-180.
Tandle A, Blazer DG 3rd, Libutti SK (2004) Antiangiogenic gene therapy of cancer: recent developments. J Transl Med 2: 22-41. 